\title{
Effect of the Rydberg states on the time evolution of nonstationary states below or just above the ionization threshold
}

\author{
Yannis Komninos ${ }^{1, *}$ and Cleanthes A. Nicolaides ${ }^{1,2, \dagger}$ \\ ${ }^{1}$ Theoretical and Physical Chemistry Institute, National Hellenic Research Foundation, 48 Vasileos Constantinou Avenue, Athens 11635, \\ Greece \\ ${ }^{2}$ Physics Department, National Technical University, Athens, Greece
}

(Received 24 May 2005; published 15 September 2005)

\begin{abstract}
We consider problems of short-time dynamics of a polyelectronic atomic nonstationary state, $V$, assumed to be formed as a wave packet at $t=0$. We focus on two cases, for which the role of the quasicontinuum of the upper part of the Rydberg states, with which the $V$ state has nonzero coupling matrix elements, is investigated. In the first case, the position of the $V$ state is just above the ionization threshold, $E=0$, and so $V$ dissipates into the free electron continuum as an autoionizing state. The question is how the presence of the Rydberg series converging to $E=0$ affects the time evolution of the autoionizing $V$. In the second case, the position of $V$ is embedded in the quasicontinuum of the Rydberg series below threshold. The question is whether there are distinct features in the time evolution of this $V$, although its position is in the discrete part of the energy spectrum. In this case, by focusing on short times and by evaluating analytically certain infinite sums, analogous to Fourier integrations, the following result is obtained: For small times, the $V$ state evolves as an exponentially decaying state. However, in addition to the term describing exponential decay, there is a term, entering with a small coefficient, which describes exponential growth and eventually dominates. It is shown that exponential decay holds for times shorter than the time $t_{p}$ needed by the wave packet to reach the outer classical turning point. For the decay to be physically meaningful, this time must be smaller than the time $t_{d}$ which equals the inverse of the half-width in atomic units. We examined a model system of $V$-Rydberg state interaction based on the Boron ${ }^{2} S$ spectrum. The results indicate that the effect is observable on the scale of femtoseconds.
\end{abstract}

DOI: 10.1103/PhysRevA.72.032716

PACS number(s): 32.80.Dz, 31.70.Hq, 31.15.-p

\section{INTRODUCTION AND DESCRIPTION OF THE PROBLEM}

The mathematical spectrum of multiparticle systems such as atoms is associated with the poles and cuts of the resolvent operator, $R(z)=1 /(z-H)$, where $z$ is a complex variable, $z=E \pm i \eta$, and $H$ is the Hamiltonian. The time evolution operator, $e^{-i H t}$, is given by

$$
e^{-i H t}=\frac{1}{2 \pi i} \oint_{\text {spectrum }} R(z) e^{-i z t} d z
$$

where the contour surrounds the spectrum of $H$ on the first Riemann sheet of $E$, in which case it is valid for $t>0$ and $t<0$. For $t>0$, e.g., the case of a nonstationary state decaying into a purely continuous spectrum irreversibly, the contour reduces to a line parallel to the real axis with $\operatorname{Im} z>0$, e.g., Refs. [1,2]. Since $R(z)$ is analytic on the first Riemann sheet, any of its possible complex poles, $z_{0}$, must appear on the second sheet. As is well known, for normal cases these are associated with resonance states-in atoms they are also called autoionizing or Auger states. In the time-dependent framework, they are associated with nonstationary states, i.e., wave packets formed at $t=0$ as localized but unstable states, and with assumed loss of information from the exci-

\footnotetext{
*Electronic address: ykomn@eie.gr

†Electronic address: caan@eie.gr
}

tation process that starts at $t=-\infty$. Let us assume the case of an isolated state. We symbolize this $t=0$ wave packet by $\Psi_{0}$ and its energy, which is real, by $E_{0}$. The square-integrable state $\left(\Psi_{0}, E_{0}\right)$ is embedded in a continuous energy spectrum of scattering states, $u_{E}$, satisfying $H_{0} u_{E}=E u_{E}$. For physical as well as mathematical reasons, the operator $H_{0}$ is given by $H_{0}=\left|\Psi_{0}\right\rangle\left\langle\Psi_{0}|H| \Psi_{0}\right\rangle\left\langle\Psi_{0}|=| \Psi_{0}\right\rangle E_{0}\left\langle\Psi_{0}\right|$ and the decay is effected by the perturbing operator $\mathrm{V}=\mathrm{H}-\mathrm{H}_{0}$. Without loss of accuracy, the spectrum of the energies $E$ into which $E_{0}$ is embedded is assumed to be the same as that defined by the full operator $H$, e.g., Refs. [1,2].

The survival probability of the wave packet is given by (with $\hbar=1$ )

$$
P(t)=\left|\left\langle\Psi_{0}\left|e^{-i H t}\right| \Psi_{0}\right\rangle\right|^{2} \equiv|G(t)|^{2},
$$

where

$$
G(t)=\sum \int g(E) e^{-i E t} d E,
$$

$g(E)$ is the distribution of $\Psi_{0}$ over the energy spectrum. The symbol of the sum in Eq. (3) stands for the discrete states below threshold. It is the role of this part of the full spectrum that the present investigation focuses on.

Once the formation of $\Psi_{0}$ is assumed, it follows from quantum mechanics that, regardless of the details of the form of the energy distribution, the probability decreases exponentially, $P(t) \approx e^{-2 \Gamma t}, t>0$ ( $\Gamma$ is the half-width), except for very short and very long times [3]. (Also see Refs. [4-7].) 


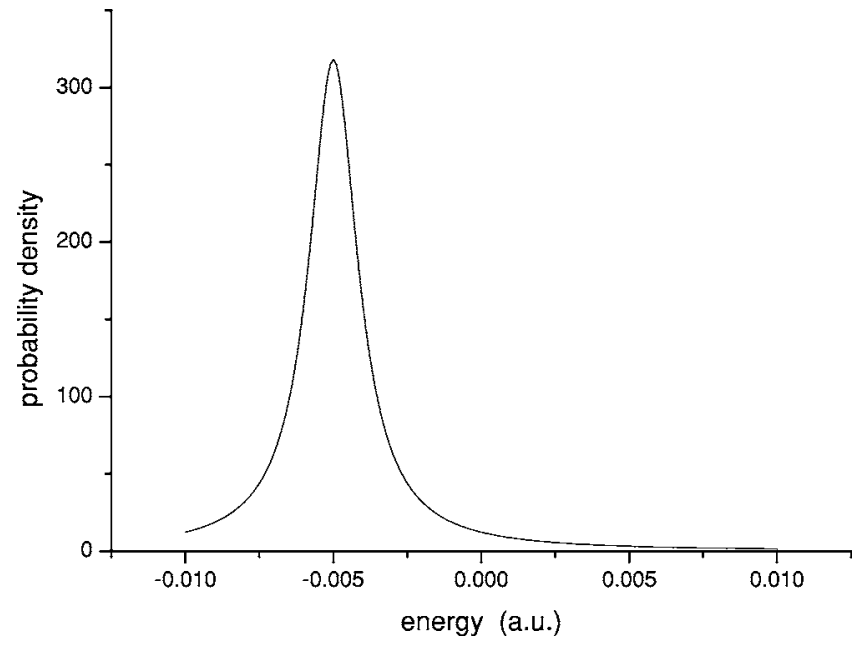

FIG. 1. A Lorentzian distribution, whose peak lies below the ionization threshold, extends mainly over the discrete part of the spectrum.

It was shown in Ref. [2] that, for the case where the self-energy function (basically the width function) is independent of energy, the mathematics of nonexponential decay (NED) can be expressed in terms of the exponential integral, $E_{1}(z)[8,9]$. Furthermore, a crucial parameter in the quantitative significance of NED is the ratio $\Gamma / E_{r}$, where $E_{r}$ is the energy of the resonance state [2,7]. The closer $E_{r}$ is to the fragmentation threshold, the quantity $\Gamma / E_{r}$ tends to an order of magnitude of unity and the importance of $E_{1}(z)$ relative to the contribution of the pole, $z_{0}$ (the cause of exponential decay), increases. The quantities $E_{1}(z)$ and the $\Gamma / E_{r}$ are used in the discussion of the sections that follow.

In all previous formal discussions of the properties of $P(t)$ for a resonance state, the spectrum which is considered in Eq. (1) is taken to be that defined exclusively by scattering states. In other words, the spectrum is continuous and there is no involvement of the discrete states below the fragmentation threshold. In atoms, the effective $-1 / r$ Coulomb potential creates an infinity of bound states, the so-called Rydberg $(R)$ states. As the ionization threshold is approached, the $R$ states form a quasicontinuum that rigorously belongs to the discrete spectrum. It is the existence of the hitherto neglected effect of the bound Rydberg states, which constitute part of the spectrum in Eq. (1), that this work has examined with respect to the time evolution of nonstationary wave packets close to threshold, below (a closed system) and above (an open system).

Specifically, two cases are examined.

(1) We assume that a very fast excitation process can create, at $t=0$, a multielectron wave packet in the form of a valence $(V)$ configuration whose $E_{0}$ is just below the ionization threshold and is embedded in the quasicontinuum of the $R$ states converging to the ionization threshold. If formation of $V$ is possible, then it must evolve by interacting with these $R$ states. In the general case, the width may cross the threshold-see Fig. 1. We ask the question: Given that the energy-dependent stationary state picture of the bound eigenfunctions produced by the $V-R$ superposition is as in Fig. 1, is there a describable time evolution of the $V$ state treated as

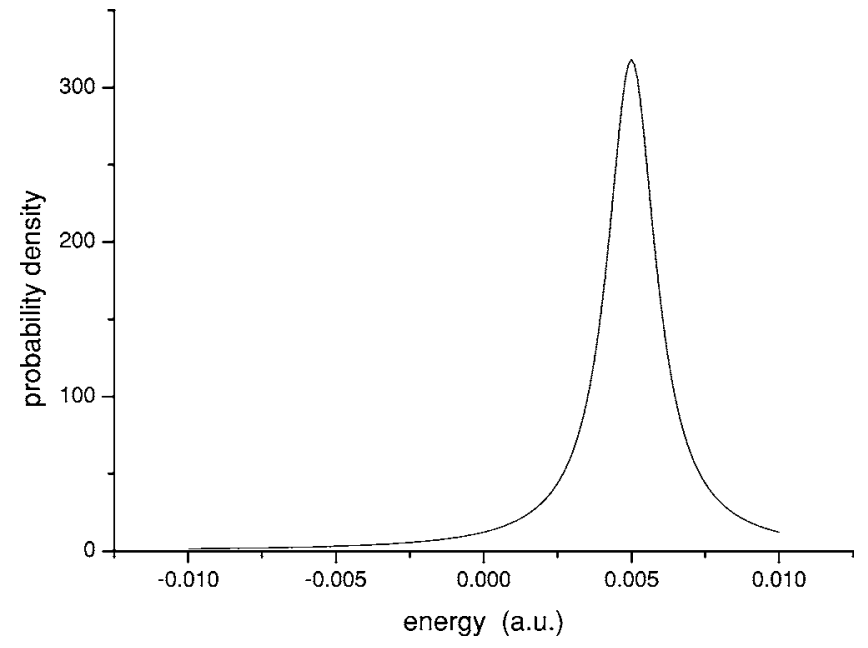

FIG. 2. A Lorentzian distribution, whose peak lies above the ionization threshold, extends mainly over the continuous part of the spectrum.

a nonstationary state, as it interacts with the $R$ states, in analogy with the case of a $\Psi_{0}$ just above threshold? Obviously, for $E_{r}$ above threshold, the system is open and the interaction of $\Psi_{0}$ with the continuum of scattering states causes autoionization and concomitant exponential decay (ED) for $P(t)$. Is there a corresponding process for the $V$ state of Fig. 1 (which defines a closed system), and for what time scales? As it will be shown in the discussion that follows, for small times (to be defined below), the $P(t)$ of the $V$ state follows ED, only this time it is succeeded by an exponential blow up as the wave packet returns from its classical turning point.

(2) Suppose that the excitation creates a nonstationary $\Psi_{0}$ and a corresponding resonance state with $E_{r}$ just above threshold, having a distribution that enters the upper part of the $R$-state discrete spectrum, as in Fig. 2. What is the effect of the Rydberg series on $P(t)$ ?

In both of the above cases, the spectral significance of the $V$ nonstationary state is diluted by the mixing with the Rydberg/continuum states. In the case of (1), it is shown that the $P(t)$ of $V$ undergoes a rapid, finite-time decay and subsequent growth, both of which are within measurable time limits. We focus on the features that emerge when the time of observation is smaller than twice the time needed for the wave packet to reach the classical outer turning point of the effective potential. In the model examined here, which is based on the Boron spectrum of ${ }^{2} S$ states, it is found that this time interval falls into the femtosecond (fs) time domain, within which the interaction produces a seemingly finite lifetime, even when the initial energy is below threshold. Such a lifetime can in principle be recorded during the ED—shown here to occur for a short period-before the $V$ state is repopulated also exponentially. In order to derive this result, we developed formulas that evaluate reliably the relevant summations, with results that resemble those derived by the corresponding integrations over the energies of the continuous part of the spectrum. 


\section{NONSTATIONARY STATE ABOVE THE IONIZATION THRESHOLD-AN OPEN SYSTEM.}

We assume the energy distribution described by Fig. 2 . The width is taken to be energy independent and, consequently, the energy distribution is a Lorentzian function. By considering that the spectrum of Eq. (1) includes the bound Rydberg series of a polyelectronic atom below the ionization threshold, the part of the distribution in the discrete spectrum has the form $[10,11]$

$$
g\left(E_{n}\right)=\frac{1}{\pi} \frac{\tau_{n} \Gamma}{\left(E_{n}-E_{r}\right)^{2}+\Gamma^{2}},
$$

where the factor $\tau_{n}=d E_{n} / d n$ ( $E_{n}$ are the energies of the perturbed $\mathrm{R}$ states) reconciles the volume normalization of states of the discrete spectrum with the energy normalization of states belonging to the continuous spectrum [11]. For practical purposes, since the $V$ state is very close to threshold, $\Gamma=\pi\left|\left\langle\Psi_{0}|V| U_{E}\right\rangle\right|^{2}$ is evaluated at the ionization threshold. For neutral atoms and positive ions, $E_{n}=-a / 2(n-\mu)^{2}$ and $\tau_{n}=a /(n-\mu)^{3}$, where $\mu$ is the quantum defect-here considered as constant - of the Rydberg series, and $a$ is a constant that depends on the units and the electric charge, the energies being measured from the ionization threshold. Upon evaluating summations over Rydberg states

$$
G_{R}(t)=\sum_{n} g\left(E_{n}\right) e^{-i E_{n} t}
$$

it is reasonable to separate a small number of lower Rydbergs, which are treated explicitly, from the infinity of the upper states which are treated approximately, as a unit. The form (4) is rewritten as

$$
g\left(E_{n}\right)=\frac{1}{2 \pi i}\left(\frac{\tau_{n}}{E_{n}-z_{0}^{*}}-\frac{\tau_{n}}{E_{n}-z_{0}}\right),
$$

where

$$
z_{0}=E_{r}-i \Gamma
$$

Here, $E_{r}=E_{0}+\Delta$, where $\Delta$ is the energy shift due to the interaction of $\Psi_{0}$ with the states of the Rydberg/continuous zero order spectrum. In the present treatment of an energyindependent self-energy, $\Delta$ is small.

Using the result of the Appendix, the part of the summation (5) over the upper Rydberg states [we write it as $\left.G_{u R}(t)\right]$, gives

$$
\begin{aligned}
G_{u R}(t) \cong & \frac{1}{2 \pi i}\left\{e^{-i z_{0} t} E_{1}\left(-i z_{0} t\right)-e^{-i z_{0} t} E_{1}\left[i\left(E_{b}-z_{0}\right) t\right]\right. \\
& \left.-e^{-i z_{0}^{*} t} E_{1}\left(-i z_{0}^{*} t\right)+e^{-i z_{0}^{*} t} E_{1}\left[i\left(E_{b}-z_{0}^{*}\right) t\right]\right\}
\end{aligned}
$$

$E_{b}$ is the energy of the $n_{b}$ th state, which is the bottom state of the upper Rydberg spectrum. This is divided between the upper and the lower Rydberg series, $G_{l R}(t)$, so that each sum includes half the contribution from the $n_{b}$ th state. The reason for this is discussed in the Appendix. Note that

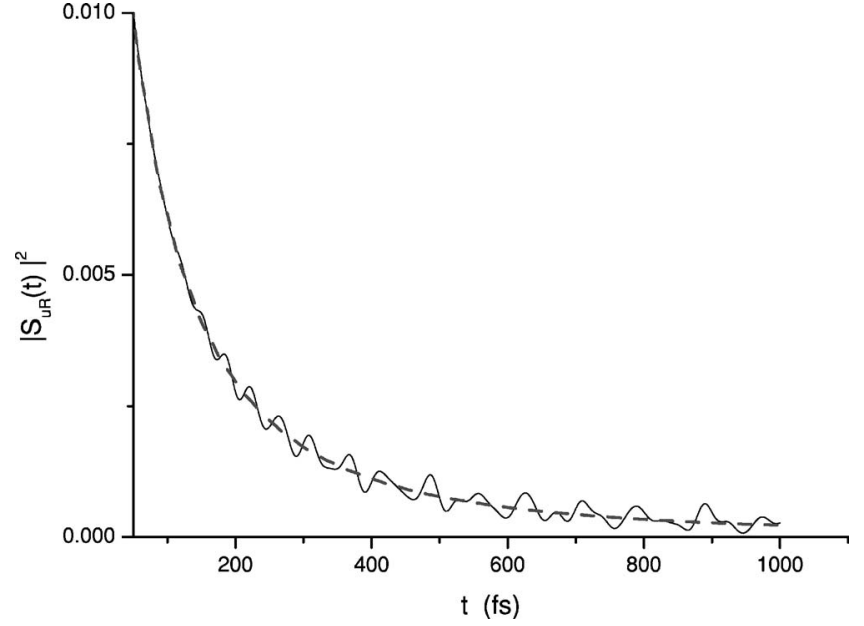

FIG. 3. The part of the survival probability, $\left|G_{u R}(t)\right|^{2}$, due to the upper Rydberg series, of an autoionizing state with $E_{r}=2$ $\times 10^{-4}$ a.u. and $\Gamma=10^{-4}$ a.u., as computed by the exact summation of Eq. (5) (continuous line) and by the sum of exponential integrals of Eq. (8) (dashed line).

$$
G_{u R}(0)=\frac{1}{\pi} \tan ^{-1}\left(\frac{\Gamma}{E_{r}}\right)-\frac{1}{\pi} \tan ^{-1}\left(\frac{\Gamma}{E_{r}-E_{b}}\right)
$$

indicating that part of the population which is missing from the continuum [the first term of Eq. (9)] resides in the upper Rydberg series. Figure 3 gives the $G_{u R}(t)$ as a function of time for $E_{r}=2 \times 10^{-4} \mathrm{a} . \mathrm{u}$. and $\Gamma=10^{-4} \mathrm{a} . \mathrm{u}$. (continuous line) to be compared with the right-hand side of Eq. (8) (dashed line). It is seen that the exponential integrals produce a kind of average of the summation for each value of $t$.

\section{NONSTATIONARY STATE BELOW THE IONIZATION THRESHOLD-A CLOSED SYSTEM}

Suppose that the $V$ state is considered as a nonstationary state, whose time evolution is caused by its interaction with the Rydberg/scattering states of the same symmetry. We resolve the Lorentzian into fractions and compute the expression

$$
G\left(z_{0}, t\right)=\sum_{n=n_{b}}^{n_{t}} \frac{\tau_{n}}{E_{n}-z_{0}} e^{-i E_{n} t}
$$

based on the results of the Appendix. The finite summation has been chosen for easy computer checks. For values of $\operatorname{Re} z_{0}>E_{t}$, which is a case analogous to that of an autoionizing state, a simple generalization of Eq. (A13) for a finite upper bound is

$$
\begin{aligned}
& \sum_{n=n_{b}}^{n_{t}} \lambda_{n b} \frac{\tau_{n}}{E_{n}-z_{0}} \lambda_{n t} e^{-i E_{n} t} \\
& \quad \simeq E_{1}\left[i\left(E_{t}-z_{0}\right) t\right] e^{-i z_{0} t}-E_{1}\left[i\left(E_{b}-z_{0}\right) t\right] e^{-i z_{0} t}
\end{aligned}
$$

where 


$$
\lambda_{n b}=\left(1-\frac{1}{2} \delta_{n n_{b}}\right) \quad \text { and } \quad \lambda_{n t}=\left(1-\frac{1}{2} \delta_{n n_{t}}\right)
$$

i.e., the first and the last term enter with coefficient 1/2. Numerical calculations show that (11) is also valid for $\operatorname{Re} z_{0}<E_{b}$, which is the case of a perturber ( $V$ state) lying inside the lower $R$ states. Thus, the result of the summation (11) is identical to that of integration according to the recipe (A15), as long as $\operatorname{Re} z_{0}$ lies outside the range of the integration. In order to evaluate the sum (11) in the case where $E_{b}<\operatorname{Re} z_{0}<E_{t}$, the equivalent of the principal value integration for sums is employed. As it has been explained elsewhere $[11,12]$, this consists of adding a cotangent term which cancels the poles of the sum. Indeed, numerical checks show that the correct formula is

$$
\begin{aligned}
& \sum_{n=n_{b}}^{n_{t}} \lambda_{n b} \frac{\tau_{n}}{E_{n}-z_{0}} \lambda_{n t} e^{-i E_{n} t}+\pi \cot \pi(\nu+\mu) e^{-i z_{0} t} \\
& \quad \simeq E_{1}\left[i\left(E_{b}-z_{0}\right) t\right] e^{-i z_{0} t}-E_{1}\left[i\left(E_{t}-z_{0}\right) t\right] e^{-i z_{0} t}-i \pi e^{-i z_{0} t}
\end{aligned}
$$

where the last term on the right-hand side is the contribution of the pole and $\nu$ is defined by the expression

$$
z_{0}=-\frac{1}{2 \nu^{2}}
$$

The discrete spectrum of the upper Rydberg states in (13) is given by $E_{n}=-1 / 2(n-\mu)^{2}$. For $t=0$, the sum in Eq. (13) is expressed in terms of gamma functions and the $\pi \cot \pi(\nu$ $+\mu)$ term arises naturally from them [11]. Setting

$$
f\left(z_{0}\right)=\frac{1}{2}[1-i \cot \pi(\nu+\mu)]
$$

Eq. (13) is written in the form

$$
\begin{aligned}
\sum_{n=n_{b}}^{n_{t}} \lambda_{n b} & \frac{\tau_{n}}{E_{n}-z_{0}} \lambda_{n t} e^{-i E_{n} t} \\
\simeq & E_{1}\left[i\left(E_{b}-z_{0}\right) t\right] e^{-i z_{0} t} \\
& \quad-E_{1}\left[i\left(E_{t}-z_{0}\right) t\right] e^{-i z_{0} t}-2 \pi i f\left(z_{0}\right) e^{-i z_{0} t} .
\end{aligned}
$$

The expression $\left(13^{\prime}\right)$ is similar to that obtained by contour integration for an autoionizing state lying in the continuum of energies above the ionization threshold. In that case, $f\left(z_{0}\right)$ equals unity while $f\left(z_{0}^{*}\right)$ equals zero in the corresponding expression $G\left(z_{0}^{*}, t\right)$ for the pole in the upper half-plane. As is shown below, this is only approximately true for energies below the ionization threshold. The implication that $f\left(z_{0}^{*}\right)$, being the coefficient of the term that blows up, is not exactly zero, is that this term will, after the initial exponential decay, dominate the time evolution.

Combination of the expressions for the two poles gives, with $E_{r}$ being the energy of $V$ below threshold,

$$
\begin{aligned}
G_{u R}(t) & \equiv \frac{1}{2 \pi i}\left[G\left(z_{0}^{*}, t\right)-G\left(z_{0}, t\right)\right] \\
& =\frac{1}{\pi_{n=n_{b}}} \sum_{n n_{b}} \frac{\tau_{n} \Gamma}{\left(E_{n}-E_{r}\right)^{2}+\Gamma^{2}} e^{-i E_{n} t} \\
& \simeq \sum E_{1}+f\left(z_{0}\right) e^{-i z_{0} t}-f\left(z_{0}^{*}\right) e^{-i z_{0}^{*} t},
\end{aligned}
$$

where the first term in the last expression signifies the sum of the contributions involving the exponential integrals [see Eq. (8)]. For $\Gamma \ll E_{r}$ the function $f\left(z_{0}\right)$ may be written in a form which illuminates the time evolution of $G_{u R}(t)$ in Eq. (16). As the behavior of the function $\cot \pi(\nu+\mu)$ in Eq. (15) is determined by $\operatorname{Im} \nu$, an expression of this quantity is required. To this purpose, the complex $\nu$ is examined,

$$
\nu \equiv\left(-2 z_{0}\right)^{-\frac{1}{2}}=\frac{1}{2\left|z_{0}\right|}\left(\sqrt{\left|z_{0}\right|-E_{r}}-i \sqrt{\left|z_{0}\right|+E_{r}}\right) .
$$

Setting $\nu_{0}=1 / \sqrt{-2 E_{r}}$, Eq. (17) is written in the form $\nu$ $=\nu_{0} F\left(\Gamma /-E_{r}\right)$ which, provided the condition $\Gamma \ll E_{r}$ is satisfied, gives

$$
\operatorname{Re} \nu \cong \nu_{0}-\frac{3}{2} \nu_{0}^{5} \Gamma^{2} \text { and } \quad \operatorname{Im} \nu \cong \nu_{0}^{3} \Gamma .
$$

Now, define the time

$$
t_{p} \equiv \pi \nu_{0}^{3}
$$

This is the half-period of the classical orbit with energy $E_{r}$ $<0$. By letting

$$
\nu_{1} \cong \nu_{0}+\mu-\frac{3}{2} \nu_{0}^{5} \Gamma^{2} \quad \text { and } \quad \mathrm{f}=\left[1-e^{-i 2 \pi \nu_{1}} e^{-2 \Gamma t_{p}}\right]^{-1},
$$

Eq. (15) gives

$$
f\left(z_{0}\right) \cong \mathrm{f} \quad \text { and } \quad f\left(z_{0}^{*}\right) \cong-\mathrm{f}^{*} e^{i 2 \pi \nu_{1}} e^{-2 \Gamma t_{p}} .
$$

Using these forms, Eq. (16) is written in the transparent form as

$$
G_{u R}(t) \cong \sum E_{1}+\mathrm{f} e^{-i \mathcal{E}_{0} t} e^{-\Gamma t}+\mathrm{f}^{*} e^{-i \mathcal{E}_{0} t} e^{i 2 \pi \nu_{1}} e^{\Gamma\left(t-2 t_{p}\right)} .
$$

Apart from very small values of $t$, the behavior of $\left|G_{u R}(t)\right|^{2}$ is determined by the last two terms which describe the contribution of the perturber, the rest being small corrections. For times smaller than the time $t_{p}$ needed for the wave packet centered on the energy $\mathcal{E}_{0}<0$ to reach the classical outer turning point, the exponential behavior dominates. For $t=t_{p}$, the two real exponentials in (22) become equal. Subsequently, the exponentially increasing term dominates and the similarity with a resonance in the continuum ends as the wave packet turns back. The right-hand side of Eq. (22) continues to provide a reasonable approximation to $|G(t)|^{2}$ as long as $t<2 t_{p}$. Defining a decay time $t_{d}=1 / \Gamma$, the condition

$$
t_{p}>t_{d}
$$

implies $e^{-2 \Gamma t_{p}} \ll 1$, and in this case the $V$ state will lose a substantial part of its population during the decay time. 


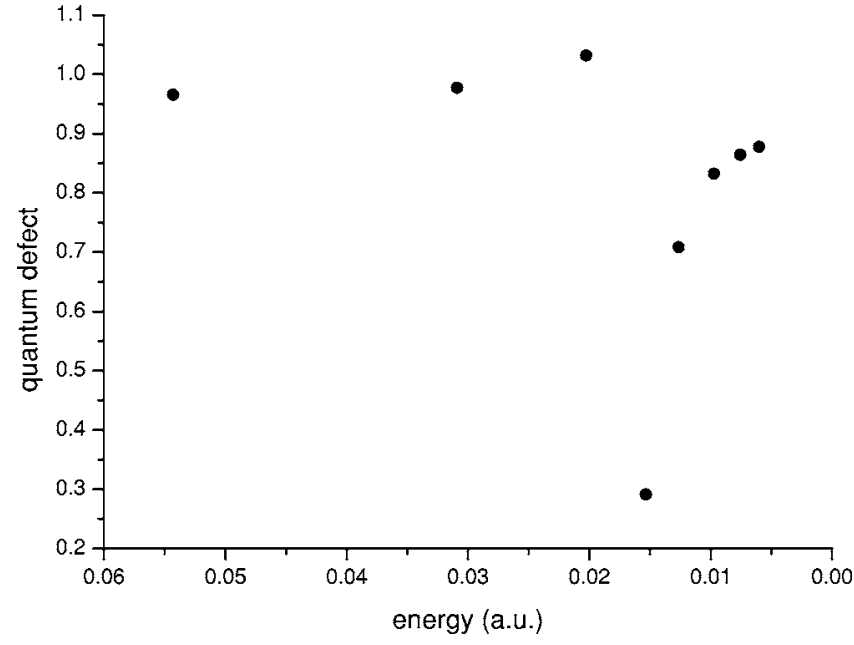

FIG. 4. Experimental quantum defects of the Boron ${ }^{2} S$ spectrum [13], exhibiting the disturbance of the Rydberg series by the $s p^{2}$ perturber.

\section{APPLICATION}

We did not find in the literature a recorded Rydberg spectrum with a $V$ state in its upper part, close to threshold. Therefore, in order to illustrate the results discussed above, we adopted a model spectrum by choosing as the $V$ state the $2 s 2 p^{2}{ }^{2} S$ valence state of Boron. In reality [13], this state is $3369 \mathrm{~cm}^{-1}$ below threshold and lies between the $6 \mathrm{~s}$ and $7 \mathrm{~s}$ Rydberg levels, after which the quasicontinuum of the ${ }^{2} S R$ states begins. The energy of the perturber is quite low $\left(t_{p}\right.$ $\approx 15 \mathrm{fs}$ ), and so the condition (23) is not fulfilled $\left(t_{d}\right.$ $\approx 242 \mathrm{fs}$ ). Thus, the exponential decay is very soon followed by an exponential growth. However, we may demonstrate the predicted effect by choosing, arbitrarily, the value of $\mathcal{E}_{0}$ to be closer to threshold. (This result would be obtained if the nuclear charge were taken to be slightly smaller than 5.) So the following approach was applied.

It is seen from experiment [13] that the ${ }^{2} S$ series has a large quantum defect (Fig. 4). This is due mainly to the penetration of the $s$ orbitals in the core. The average value $\mu$ $=0.88$ was chosen for the problem. What we did is to assume that the position of the $V$ state is higher (and therefore embedded in the quasicontinuum of the Rydberg series), and to compute its interaction with the state at the ionization threshold using Hartree-Fock wave functions. (For this symmetry, electron correlation is not important as far as the problem of interest is concerned.) Such a computation provides the required quantity, $\Gamma \sim 10^{-4}$ a.u.

The exponential decay is indeed obtained, as is depicted in Figs. 5(a)-5(c), where $\left|G_{u R}(t)\right|^{2}$ is plotted as a function of time for $\nu_{0}=15,17,19$ with $t_{p} \approx 256.5,373.3,521.2 \mathrm{fs}$, respectively. The exact summation is given by the continuous line, while the analytic formula [right-hand side of Eq. (22)] is given by the dashed line. The latter describes the evolution of the wave packet during the first cycle only.

\section{CONCLUSION}

Given the current possibilities of observing processes occurring within very short time scales, we considered that it
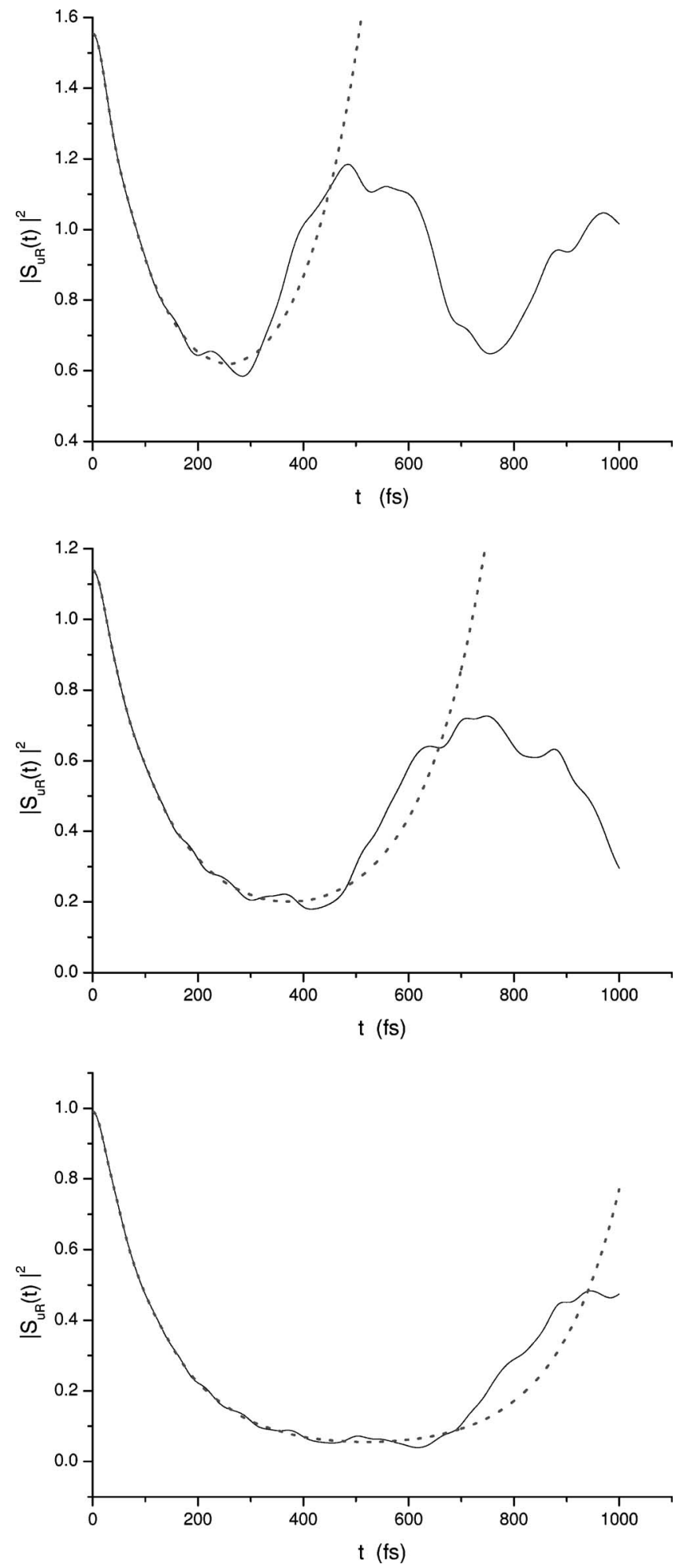

FIG. 5. The part of the survival probability, $\left|G_{u R}(t)\right|^{2}$, of a perturber state with $\mathcal{E}_{0}=-1 / 2 \nu_{0}^{2}$ for $\nu_{0}=15,17$, and 19 and $\Gamma$ $=10^{-4}$ a.u., as computed by the exact summation of Eq. (5) (continuous line) and by the analytic form of Eq. (22) (dashed line).

would be useful to examine the fundamentals of the time evolution not only of nonstationary states that are part of the continuous spectrum but also of specific cases of polyelectronic states belonging to the discrete spectrum of atoms. In 
such spectra, a localized wave packet, which is labeled by a valence configuration, $V$, may be prepared at $t=0$ close to threshold, either above or below. If the state $V$ is in the continuous spectrum, it dissipates by emitting an electron. However, if it is below threshold, then it is part of the discrete spectrum, and the accepted wisdom is that its time dependence is a sum of phase factors, $e^{i E_{n} t}$, involving eigenvalues, $E_{n}$.

In the present work, we showed how the existence of Rydberg series affects the dynamics of states of both categories. The interesting finding is the fact that, under special conditions, the survival probability of the $V$ state in the discrete spectrum may exhibit, for a short period of time, exponential decay which, in principle, is observable, provided such states exist and experiments manage to prepare them and measure their short-time evolution.

\section{ACKNOWLEDGMENTS}

This work was supported by the program "Pythagoras" which is co-funded by the European Social Fund (75\%) and National Resources (25\%), Operational Program for Educational and Vocational Training II (EPEAEK II).

\section{APPENDIX}

The sum

$$
G_{u R}\left(z_{0}, t\right)=\sum_{n=n_{b}}^{\infty} \frac{\tau_{n}}{E_{n}-z_{0}} e^{-i E_{n} t}
$$

which refers to the upper Rydberg series, is evaluated below. To this purpose, use will be made of one of the addition theorems of the confluent hypergeometric (Kummer's) function $U(a ; c ; z)$. This is a multiple-valued function with its principal branch at $-\pi<\arg z \leqslant \pi$ and the addition theorem of interest is [9]

$$
e^{z^{\prime}} \sum_{q=0}^{\infty} \frac{(-1)^{q}}{q !}\left(z^{\prime}\right)^{q} U(a ; c+q ; z)=U\left(a ; c ; z+z^{\prime}\right) .
$$

Setting both parameters $a$ and $c$ equal to unity, and using the relation of the resulting special form to the incomplete gamma function, namely

$$
U(1 ; q+1 ; z)=z^{-q} e^{z} \Gamma(q, z),
$$

Eq. (A2) becomes

$$
\sum_{q=0}^{\infty} \frac{(-1)^{q}}{q !}\left(\frac{z^{\prime}}{z}\right)^{q} \Gamma(q, z)=\Gamma\left(0, z+z^{\prime}\right)
$$

Furthermore, since the exponential integral is a special form of the incomplete gamma function,

$$
\Gamma(0, z)=E_{1}(z),
$$

Eq. (A4) can be written as

$$
\sum_{q=1}^{\infty} \frac{(-1)^{q}}{q !}\left(\frac{z^{\prime}}{z}\right)^{q} \Gamma(q, z)=E_{1}\left(z+z^{\prime}\right)-E_{1}(z)
$$

where the infinite summation now starts from unity. Finally, since $(\partial / \partial z) E_{1}(z)=-e^{-z} / z$, differentiation of Eq. (A6) with respect to $z^{\prime}$ produces the formula

$$
\sum_{q=1}^{\infty} \frac{(-1)^{q}}{(q-1) !}\left(\frac{z^{\prime}}{z}\right)^{q} \Gamma(q, z)=-\frac{z^{\prime}}{z+z^{\prime}} e^{-\left(z+z^{\prime}\right)} .
$$

Equations (A6) and (A7) are extremely useful in evaluating the sum of the upper Rydberg states. Specifically, (A7) is used to represent the $n$th term of (A1) as a power series expansion about the threshold $\left(E_{\mathrm{th}}=0\right.$ in our choice). Setting $z^{\prime}=i E_{n} t$ and $z=-i z_{0} t$ in (A7) results in

$$
\frac{-E_{n}}{E_{n}-z_{0}} e^{-i E_{n} t}=\sum_{q=1}^{\infty} \frac{(-1)^{q}}{(q-1) !}\left(\frac{-E_{n}}{z_{0}}\right)^{q} \Gamma\left(q,-i z_{0} t\right) e^{-i z_{0} t}
$$

To proceed further, the functional form of the energy is specified, $E_{n}=-1 / 2(n-\mu)^{2}$, where $\mu$ is the quantum defect considered as constant for the members of the upper Rydberg series. Then, $\tau_{n}=1 /(n-\mu)^{3}$. Multiplying (A8) by $2 /(n-\mu)$ and summing with respect to $n$, in order to evaluate (A1), we obtain an expression in which the summation over $n$ has been transformed to a summation over $q$,

$$
\begin{aligned}
\sum_{n=n_{b}}^{\infty} \frac{\tau_{n}}{E_{n}-z_{0}} e^{-i E_{n} t}= & 2 \sum_{q=1}^{\infty} \frac{(-1)^{q}}{(q-1) !} \frac{\zeta\left(2 q+1, n_{b}-\mu\right)}{\left(2 z_{0}\right)^{q}} \Gamma(q, \\
& \left.-i z_{0} t\right) e^{-i z_{0} t},
\end{aligned}
$$

where use has been made of the generalized Riemann zeta function

$$
\zeta\left(2 q+1, n_{b}-\mu\right) \equiv \sum_{n=0}^{\infty} \frac{1}{\left(n+n_{b}-\mu\right)^{2 q+1}}=\sum_{n=n_{b}}^{\infty} \frac{1}{(n-\mu)^{2 q+1}} .
$$

For large values of the argument $n_{b}-\mu$, the generalized Riemann zeta function is approximated by the asymptotic series [9]

$$
\begin{aligned}
\zeta\left(2 q+1, n_{b}-\mu\right) \sim & \frac{1}{(2 q) !}\left(\frac{(2 q-1) !}{\left(n_{b}-\mu\right)^{2 q}}+\frac{(2 q) !}{2\left(n_{b}-\mu\right)^{2 q+1}}\right. \\
& \left.+\sum_{k=1}^{m} B_{2 k} \frac{(2 k+2 q-1) !}{(2 k) !\left(n_{b}-\mu\right)^{2 k+2 q}}\right) . \quad \text { A }
\end{aligned}
$$

Apart from the second term, the asymptotic series for $\zeta\left(2 q+1, n_{b}-\mu\right)$ contains only even powers and is easily written in terms of the energy $E_{b}$. Switching to energies instead of quantum numbers results in the approximation 
$\zeta\left(2 q+1, n_{b}-\mu\right) \sim \frac{\left(-2 E_{b}\right)^{q}}{2 q}+\frac{1}{2}\left(-2 E_{b}\right)^{q+\frac{1}{2}}+O\left[\left(-2 E_{b}\right)^{q+1}\right]$.

The value of $n_{b}$ must be such that the error remains sufficiently small in the worst case $q=1\left(n_{b}=\ell+5\right.$ is a reasonable choice). Setting the expression (A12) in Eq. (A9) we make use of Eqs. (A6) and (A7) in order to evaluate the sum over $q$. The second term of (A12) results in an expression equal to half the first term of the summation (A9). Thus, in order to keep things simple, we redefine the original summation so that it contains half of the state $n_{b}$, the other half being included in the summation of the lower Rydberg states, specifically

$$
\sum_{n=n_{b}}^{\infty} \lambda_{n b} \frac{\tau_{n}}{E_{n}-z_{0}} e^{-i E_{n} t} \cong E_{1}\left[i\left(E_{b}-z_{0}\right) t\right] e^{-i z_{0} t}-E_{1}\left(-i z_{0} t\right) e^{-i z_{0} t},
$$

where $\lambda_{n b}=1-\frac{1}{2} \delta_{n b}$. Unfortunately, the region of validity of the expansion (A2) is not indicated in the mathematical handbooks. Numerical computation shows that (A13) is a good approximation for $\operatorname{Re} z_{0}>0$ and for times

$$
\operatorname{Re}\left(z_{0}\right) t=O(1)
$$

Thus, for not too large values of $t$, the sum of the upper Rydbergs results into the two exponential integrals the second of which cancels a similar term arising from the integration over the continuous spectrum. The result is identical to the one obtained by treating the infinity of the upper states as an appendage of the continuous spectrum, i.e., the summation is replaced by an integration according to the usual recipe

$$
\sum_{n} \tau_{n} f(n) \approx \int \tau_{n} f(n) d n=\int f(E) d E
$$

where $\tau_{n}=\partial E_{n} / \partial n$. In the above treatment this procedure is justified and the approximations made are explicitly stated. Moreover, correction terms may be employed whenever they are needed.
[1] M. L. Goldberger and K. M. Watson, Collision Theory (Wiley, New York, 1964).

[2] C. A. Nicolaides and D. R. Beck, Int. J. Quantum Chem. 14, 457 (1978); Phys. Rev. Lett. 38, 683 (1977).

[3] The issue of the possible violation of exponential decay (ED) of isolated states has been attracting the attention of scientists for many decades. For reviews with different emphases see Refs. $[1,2,4]$. The fundamental discussion of Goldberger and Watson [1] on this problem employs the powerful formalism of the resolvent operator, on which later work was based $[2,6]$. For additional analysis and for $a b$ initio calculations regarding real atomic systems (and not models), see Refs. [2,5-7]. References $[1,2,4-6]$ cite a large number of papers on non-ED.

[4] H. Nakazato, M. Namiki, and S. Pascazio, Int. J. Mod. Phys. B 10, 247 (1996).

[5] Th. Mercouris and C. A. Nicolaides, Phys. Rev. A 65, 012112 (2002); C. A. Nicolaides and Th. Mercouris, J. Phys. B 29, 1151 (1996); Th. Mercouris and C. A. Nicolaides, ibid. 30,
811 (1997).

[6] C. A. Nicolaides, Phys. Rev. A 66, 022118 (2002).

[7] T. G. Douvropoulos and C. A. Nicolaides, Phys. Rev. A 69, 032105 (2004).

[8] Handbook of Mathematical Functions edited by M. Abramowitz and I. A. Stegun (Dover, New York, 1965).

[9] W. Magnus, F. Oberhettinger, and R. P. Soni, Formulas and Theorems for the Special Functions of Mathematical Physics (Springer-Verlag, New York, 1966).

[10] Y. Komninos and C. A. Nicolaides, Phys. Rev. A 70, 042507 (2004).

[11] Y. Komninos, G. Aspromallis, and C. A. Nicolaides, J. Phys. B 28, 2049 (1995).

[12] Y. Komninos and C. A. Nicolaides, J. Phys. B 37, 1817 (2004).

[13] Atomic Energy Levels, edited by C. E. Moore (National Bureau of Standards, Washington, DC, 1949), circular 467. 